\title{
Kainic Acid Lesions of the Dorsal Nucleus of the Lateral Lemniscus: Effects on Binaural Evoked Responses in Rat Auditory Cortex
}

\author{
S. Lynn Glenn and Jack B. Kelly \\ Laboratory of Sensory Neuroscience, Psychology Department, Carleton University, Ottawa, Ontario K1S 5B6, Canada
}

The effects of unilateral lesions of the dorsal nucleus of the lateral lemniscus (DNLL) on binaural processing were examined by measuring the amplitude of auditory cortical evoked responses in the albino rat. Lesions were made by pressure injection of small quantities of kainic acid through a micropipette lowered into the lateral lemniscus. Comparisons were made between normal animals and animals with unilateral DNLL damage. In normal animals, the amplitude of evoked potentials recorded from left and right primary auditory cortex varied as a function of the time difference between clicks delivered to the two ears. Maximum responses were obtained from the hemisphere contralateral to the leading click, and response amplitude was progressively reduced as the interaural time difference (ITD) was shifted in favor of the ipsilateral ear over the range from $+600 \mu \mathrm{sec}$ to $-600 \mu \mathrm{sec}$. The functions in the left and right hemisphere were symmetrical mirror images of one another. Destruction of the DNLL had no significant effect on the maximum response amplitude, evoked response threshold, or response latency in either hemisphere. On the other hand, the lesion did have the effect of greatly reducing the slope of the ITD function in the hemisphere contralateral to the lesion. The change in slope was attributed to a reduction in the strength of inhibition produced by stimulation of the ipsilateral ear. No effect was seen on the slope of the function in the ipsilateral hemisphere. Animals with lesions that spared DNLL but destroyed the intermediate and ventral nucleus of the lateral lemniscus had normal binaural response functions. These data show that the DNLL plays an important role in shaping binaural responses in the contralateral auditory pathway.

Several lines of evidence suggest that the dorsal nucleus of the lateral lemniscus (DNLL) plays a role in shaping binaural responses in the central auditory system. First, the majority of neurons in DNLL are sensitive to binaural stimulation. Both binaural summation and binaural suppression responses can be recorded from DNLL (Aitkin et al., 1970; Brugge et al., 1970). Most binaural neurons are activated preferentially by sounds representing positions in the contralateral spatial field. Thus,

Received Feb. 20, 1992; revised Apr. 10, 1992; accepted Apr. 21, 1992.

This research was supported by an operating grant from the Natural Sciences and Engineering Research Council of Canada. We thank Joan Mallet for technical assistance in preparation of photomicrographs.

Correspondence should be addressed to Jack B. Kelly, Ph.D., at the above address.

Copyright (C) 1992 Society for Neuroscience $0270-6474 / 92 / 123688-12 \$ 05.00 / 0$ the physiological responses in DNLL are lateralized with regard to binaural stimulation in much the same way as responses in more rostral auditory structures, such as inferior colliculus (IC) and auditory cortex. Second, the DNLL is the source of decussating fibers through the commissure of Probst to the contralateral DNLL and IC (Goldberg and Moore, 1967; Beyerl, 1978; Adams, 1979; Brunso-Bechtold et al., 1981; Glendenning et al., 1981; Kudo, 1981; Zook and Casseday, 1982, 1987; Coleman and Clerici, 1987; Shneiderman et al., 1988; Hutson et al., 1991). This pathway might provide an important anatomical substrate for neural interactions related to laterality of spatial responses in the auditory system. Most of the neurons in DNLL are GABAergic (Adams and Mugnaini, 1984; Thompson et al., 1985; Moore and Moore, 1987; Roberts and Ribak, 1987; Glendenning and Baker, 1988), and the projections to the contralateral IC and DNLL are characterized by terminals with pleomorphic vesiclcs and symmetrical synaptic contacts (Oliver and Shneiderman, 1989; Shneiderman and Oliver, 1989). Thus, one would expect the DNLL to exert a net inhibitory influence on neural responses on the opposite side of the brain. The orderly distribution of GABAergic terminals along isofrequency laminae in the IC and the interdigitation of these terminals with potentially excitatory terminals arising from other sources suggest that the DNLL may play a role in establishing contralateral dominance of neural responses in the central auditory system (Shneiderman et al., 1988). Third, physiological evidence indicates that local application of the $\mathrm{GABA}_{\mathrm{A}}$ antagonist bicuculline can dramatically alter binaural response characteristics of neurons in the central nucleus of the IC (Faingold et al., 1989, 1991). These data imply that binaural responses are influenced by inhibitory interactions occurring within the $\mathrm{IC}$ itself. The most likely source of this inhibition is the contralateral DNLL.

The present study was undertaken to evaluate the contribution of DNLL to binaural responses in the auditory pathway. Auditory cortical evoked responses were used as an index of binaural sensitivity in normal animals and animals with unilateral kainic acid lesions of the DNLL. In the normal rat, the amplitude of the cortical evoked response is sensitive to small time differences between paired clicks delivered separately to the two ears. The response amplitude is larger in the hemisphere contralateral to the ear that is stimulated first and varies systematically with manipulation of interaural time differences (ITDs) over the range between +600 and $-600 \mu \mathrm{sec}$. The size of the cortical response can be reduced by half as the ITD is shifted in favor of the ipsilateral ear. Comparison of cortical responses in normal and brain-damaged animals allowed an assessment of the contribution of DNLL to binaural sensitivity ipsilateral and contralateral to the lesion. 


\section{Materials and Methods}

Subjects. Successful electrophysiological studies were completed on 17 male Wistar rats (Rattus norvegicus) obtained from Charles River, Inc. (St. Constant, Quebec). All animals were between 250 and $500 \mathrm{gm}$ at the time of physiological recording. Otoscopic examination revealed no obstruction or abnormality of the external meatus or tympanic membrane in any animal. Five of the 17 rats received unilateral kainic acid lesions of the DNLL at least 2 weeks prior to the recording session. Three received lesions of the right DNLL (R167, R168, and R173), and two received lesions of the left DNLL (R171 and R178). Three animals (R162, R175, and R165) received lesions that spared the DNLL but damaged neighboring auditory structures including the ventral (VNLL) and intermediate (INLL) nuclei of the lateral lemniscus or the inferior colliculus. Three other animals (R174, R164, and R170) underwent surgery, including insertion of a glass micropipette into DNLL for delivery of the kainic acid, but no lesion was made. In each of the animals with kainic acid lesions or insertion of the micropipette, the posterior neocortex was aspirated on one side of the brain to facilitate access to the auditory brainstem.

Six additional animals served as normal controls. For these animals, the pipette was not inserted and no kainic acid injection was made. In five of the six cases, however, the posterior neocortex was aspirated to control for possible effects of cortical ablation. Three cases received left (R172, R182, and R189) and two received right (R163 and R179) aspiration of the posterior neocortex. The remaining animal (R 169) had no surgical treatment.

Kainic acid lesions. In preparation for kainic acid lesions, the animals were deeply anesthetized with Equithesin $(3 \mathrm{ml} / \mathrm{kg}$, i.p.). Equithesin was prepared from $21.3 \mathrm{gm}$ of chloral hydrate and $4.8 \mathrm{gm}$ of sodium pentobarbital dissolved in $1.5 \%$ benzyl alcohol, $40 \%$ propylene glycol, $10 \%$ ethanol, $2.5 \%$ sodium benzoate, $2.5 \%$ benzoic acid, and $43.5 \%$ distilled $\mathrm{H}_{2} \mathrm{O}$ to make $500 \mathrm{ml}$ of solution. The $\mathrm{pH}$ was adjusted to 6.7 using sodium hydroxidc. Atropine sulfate $(0.05 \mathrm{mg} / \mathrm{kg}$, i.p.) was administered prior to surgery to prevent respiratory distress. The animal was then placed in a head holder that left the ear canals free so that damage to the tympanic membranes could be avoided. Following a midline incision and retraction of the scalp, a wide craniotomy was performed to expose the posterior neocortex. The caudal pole of the neocortex was then aspirated to expose the ventrolateral portion of the inferior colliculus.

Kainic acid was dissolved in Locke's solution $(1 \mathrm{mg} / \mathrm{ml})$ and delivered into the DNLL through a glass micropipette with a tip diameter of $60-$ $80 \mu \mathrm{m}$. The pipette was inserted into the DNLL with a micromanipulator. The micropipette was connected via Sylastic and polyethylene tubing to a $10 \mu 1$ Hamilton syringe. The syringe and tubing were filled with distilled water, and the pipette tip was filled with kainic acid. After the pipette was positioned in the brain, $1.5-2 \mu 1$ (up to $15 \mathrm{nmol}$ ) of kainic acid were pressure injected with a syringe pump at the rate of $1.5 \mathrm{nmol} / \mathrm{min}$. To allow for diffusion of the kainic acid into the surrounding tissues, the micropipette was left in place for approximately 5-10 min. Gelfoam, moistened with saline, was used to cover the exposed tissue, and the scalp was sutured in place. Approximately $45 \mathrm{~min}$ following surgery, diazepam $(4.5 \mathrm{mg} / \mathrm{kg}$, i.p.) was administered as a prophylactic against seizure activity.

Physiological recordings. Following a recovery period of at least 2 weeks, the animals were prepared for recording from the auditory cortex. Anesthesia was induced with Equithesin $(3 \mathrm{ml} / \mathrm{kg}$, i.p.) and maintained with supplemental doses $(0.5 \mathrm{ml} / \mathrm{kg}$, i.p.) administered approximately every $45 \mathrm{~min}$ to achieve a state of areflexia throughout the recording session, which typically lasted $10-12 \mathrm{hr}$. Body temperature was held constant at $37^{\circ} \mathrm{C}$ with a hot-water heating pad. The animal's head was secured in a head holder, leaving the ears unobstructed for the insertion of acoustic couplers and headphone drivers. Following a midline incision and retraction of the scalp, a wide craniotomy was performed to expose the auditory cortex on both sides of the brain.

The animal was then transported to an electrically shielded, soundattenuated room and earphones were positioned in the left and right external meatuses for delivery of paired click stimuli. Evoked potentials were recorded with silver ball electrodes placed on the surface of the dura mater overlying primary auditory cortex. Potentials were amplified $10,000 \times$, bandpass filtered $(0.3-10,000 \mathrm{~Hz}$ ), and averaged on line (Grass model P511 amplifier and Tracor Northern model 1550 signal averager). Evoked responses were determined from an average of 50 single sweeps. Thresholds for eliciting an averaged evoked response by monaural con-

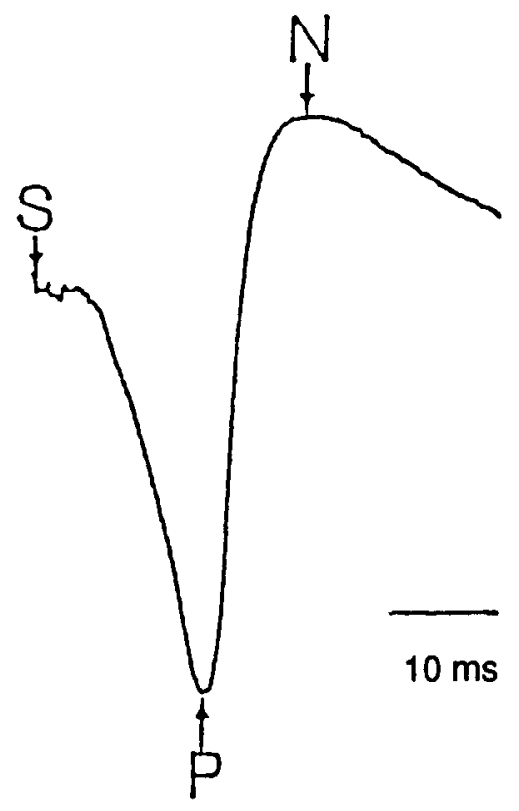

Figure 1. An auditory cortical evoked response to clicks presented binaurally with an interaural time delay of $-400 \mu \mathrm{sec}$ (left leading right ear stimulation). The response was recorded from the right primary auditory cortex and is based on an average of 50 stimulus presentations. The response is characterized by a large positive deflection $(P)$ followed by a negative deflection $(N)$ with the cortical electrode referenced to an electrode inserted between the scalp and skull at the rostral margin of the midline incision. The latency of the response was measured from stimulus onset $(S)$ to the positive peak $(P)$, and the amplitude was obtained by measuring the difference in microvolts between the positive peak $(P)$ and the immediately following negative peak $(N)$ as indicated by the arrows. The peak-to-peak amplitude of this response was $63 \mu \mathrm{V}$.

tralateral stimulation were determined for left and right hemispheres by lowering the sound pressure level (SPL) until no response was detected. The sound pressure was then fixed at approximately $40 \mathrm{~dB}$ above threshold in both ears and amplitude and latency measures were obtained.

Amplitude and latency measurements are illustrated in Figure 1, which shows a typical averaged evoked response from the rat's primary auditory cortex. The response is characterized by a large positive (downward) deflection labeled "P" on the sample waveform followed by a negative (upward) excursion labeled "N." The latency of the response was measured from stimulus onset to the first positive peak, and the amplitude was obtained by measuring the difference in microvolts between the first positive peak and the immediately following negative peak as indicated by the arrows.

Stimulus conditions. Clicks were produced by passing square waves, $100 \mu \mathrm{sec}$ in duration, through two passive decade attenuators (Hewlett Packard, model 350D) and a power amplifier to headphone drivers mounted in customized housings (Pioneer stereo headphone driver SE50D). Fach headphone housing was connected to a sealed acoustic coupler with an open speculum that could be inserted into the rat's external meatus. The couplers were constructed so that a probe tube could be inserted for acoustical measurements along the length of the speculum with its tip centered at the open end within a few millimeters of the tympanic membrane. Maximum click intensity was determined at the earphone opening with a measuring amplifier (Bruel and Kjaer, model 2218) and a $1 / 2$ inch condenser microphone (Bruel and Kjaer, model 4134) connected to the probe tube assembly. The stimulus repetition rate was $2 / \mathrm{sec}$.

A series of binaural clicks was presented with ITDs ranging from $-600 \mu \mathrm{sec}$ to $+600 \mu \mathrm{sec}$. The order of presentation was randomized according to a predetermined schedule. The ITD values were \pm 600 , $\pm 500, \pm 400, \pm 300, \pm 200, \pm 150, \pm 100, \pm 75, \pm 50, \pm 25$, and $0 \mu \mathrm{sec}$. These values were selected on the basis of previous experiments with normal animals to provide a detailed description of the amplitude changes around $0 \mu \mathrm{sec}$. The entire sequence of ITD values was repeated 10 times 

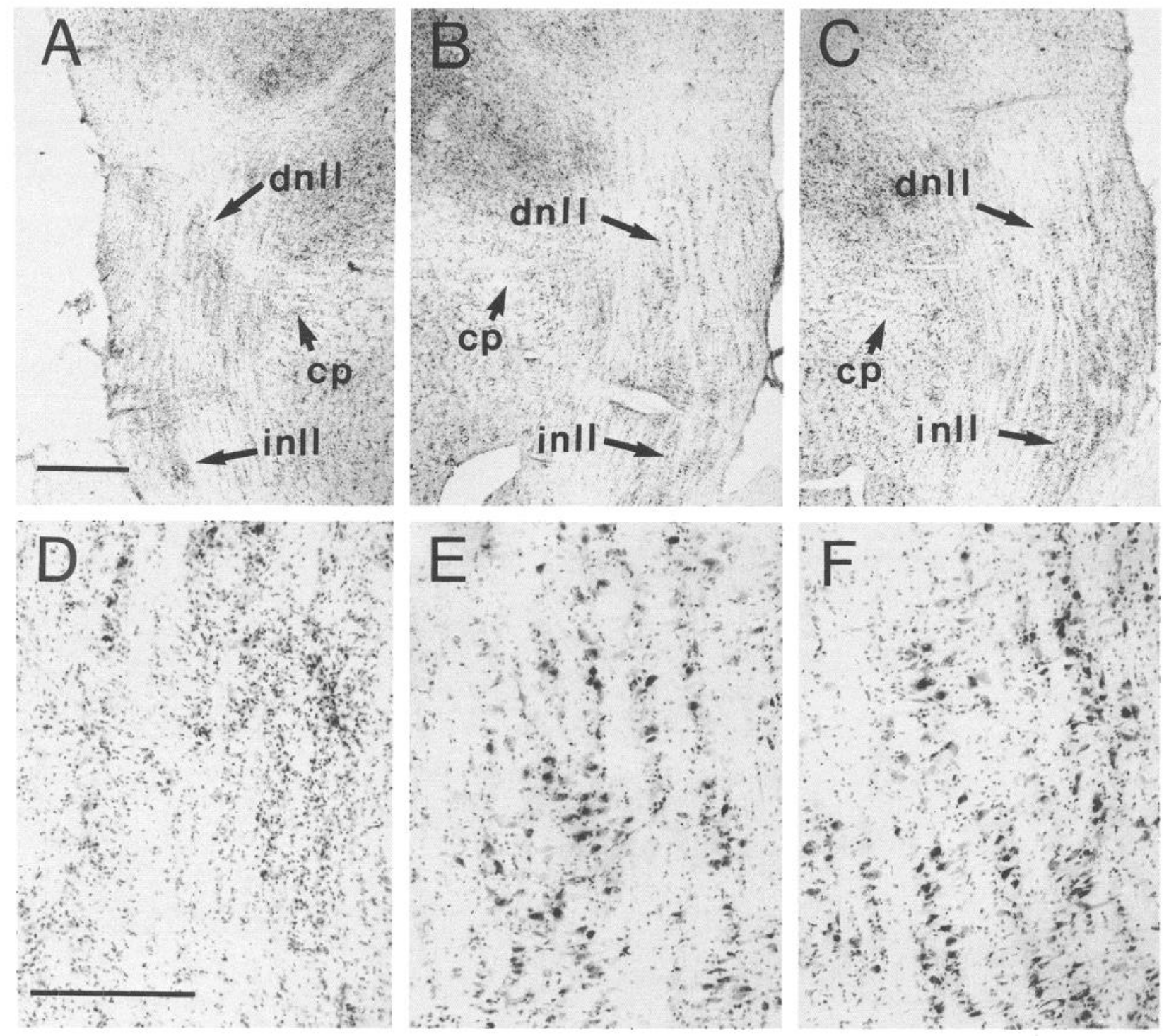

Figure 2. Photomicrographs of cresyl violet-stained frontal sections taken through the brainstem of an animal with a left DNLL lesion (R178). $A$ shows the extent of cell loss in DNLL on the left side of the brain. $B$ shows the DNLL on the right side of the brain in the same animal. $C$ shows a corresponding frontal section through the right DNLL of a normal animal (R179). $D-F$ are higher-power photomicrographs taken from DNLL as delineated in $A-C$, respectively. Nerve cell loss is apparent throughout the left DNLL $(D)$, but no cell loss is seen in the right DNLL $(E)$. The cell loss illustrated in $D$ can also be compared to cells in a normal DNLL $(F)$. c $p$, commissure of Probst; $d n l l$, dorsal nucleus of the lateral lemniscus; inll, intermediate nucleus of the lateral lemniscus. Scale bars: $A, 500 \mu \mathrm{m}$ for $A-C ; D, 200 \mu \mathrm{m}$ for $D-F$.

to control for possible changes in the response amplitude over time. Therefore, the data obtained from each animal consisted of 10 blocks of trials, each of which covered the full range of ITD values. For ease of comparison of data from different animals, the evoked response amplitudes were normalized and expressed as a percentage of the maximum response obtained from each hemisphere. The mean amplitude was plotted as a function of interaural time differences for each animal. The ITD response amplitude functions for animals with DNLL lesions were compared with those from normal control and lesion control cases.

Histological Procedures. Following the recording sessions, the animals were deeply anesthetized with Equithesin and perfused through the heart with saline and $10 \%$ formalin. The brains were then immersed in formalin, sectioned at $50 \mu \mathrm{m}$ in the frontal plane, and stained with cresyl violet to examine the extent of the lesions. The extent of damage was plotted for each case with reference to a standard series of sections through the auditory midbrain. The extent of cell loss was ranked in comparison with normal animals as either complete (96-100\%), severe (76-95\%), moderate $(46-75 \%)$, or mild (16-45\%). Tissue with cell loss less than $15 \%$ was considered normal. The extent of damage was illustrated at $200 \mu \mathrm{m}$ intervals through the DNLL.

\section{Results}

Kainic acid lesions. Kainic acid lesions destroyed nerve cell bodies in the vicinity of the injection site with varying degrees of cell loss. Lesions were often accompanied by intense gliosis in the area damaged by the injection. Cell loss was restricted to the vicinity of the cannula tip, and there was no indication of damage to areas remote from the target site except for cases in which the cannula tip was inadvertently misplaced. A typical DNLL lesion is shown in Figure 2.

Anatomical reconstructions are presented in Figure 3 for three rats (R167, $\mathrm{R} 168$, and $\mathrm{R} 173$ ) with right DNLL lesions. In two of these cases (R167 and R168), DNLL suffered moderate to mild cell loss throughout its rostral to caudal extent. The INLL, VNLL, and IC were not damaged in these cases. In the third rat (R173), DNLL had moderate to severe damage. The INLL and VNLL were also damaged. 

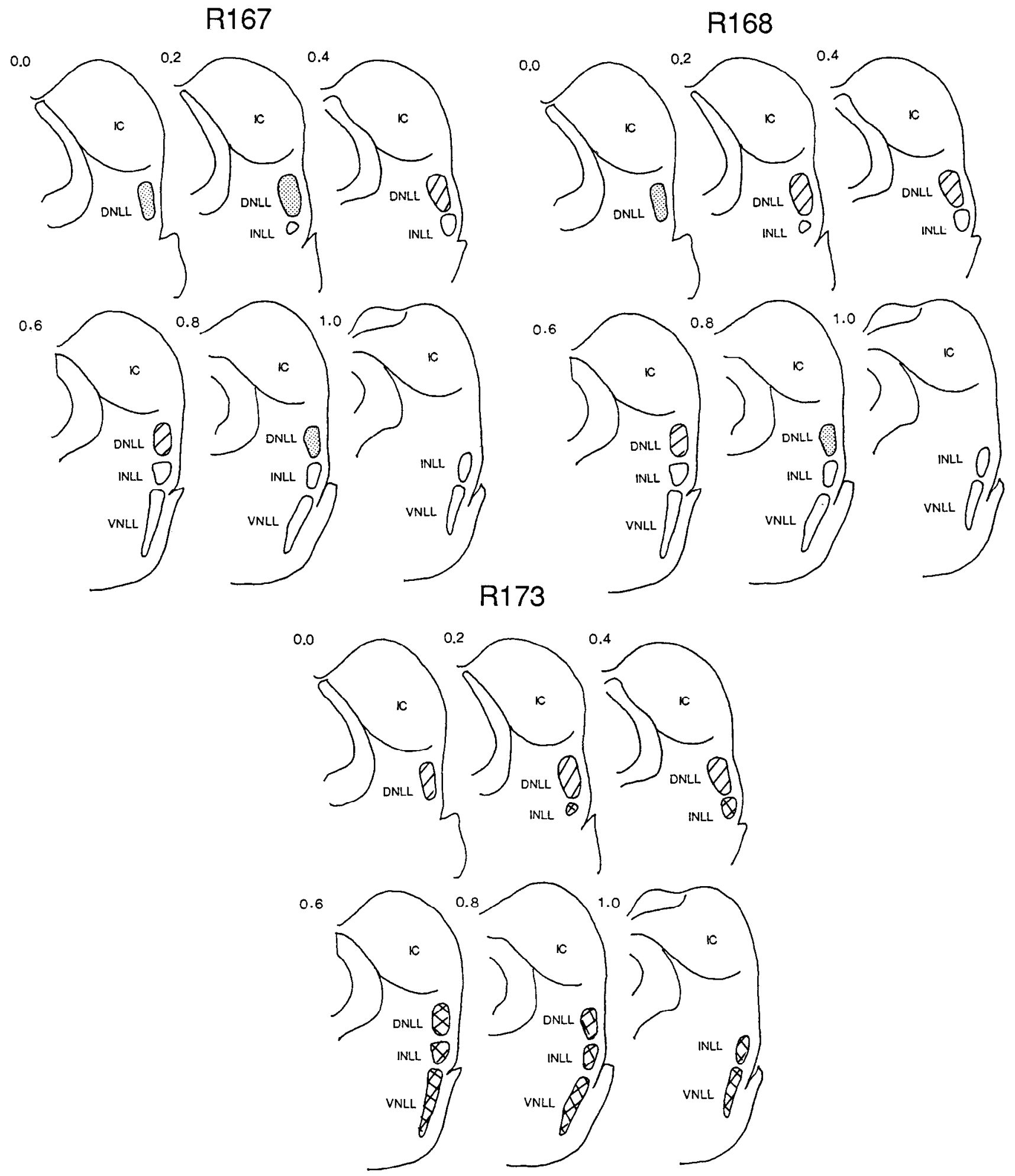

Figure 3. Anatomical reconstructions for three animals $(R 167, R 168$, and $R 173)$ with right DNLL lesions. Standard frontal sections represent $200 \mu \mathrm{m}$ intervals through the lateral lemniscus. For Figures 3-5, dark shading (Figs. 4, 5), cross-hatching, hatching, and light shading indicate complete, severe, moderate, and mild cell loss, respectively.

Anatomical reconstructions for two rats with left DNLL lesions (R171 and R178) are shown in Figure 4. In the case of R171, DNLL exhibited moderate to mild cell loss, while INLL and VNLL were completely spared. In the case of R178, the injection resulted in severe to complete cell loss in DNLL. The
INLL, VNLL, and a small portion of the ventral IC were also damaged.

In three cases the kainic acid lesions failed to damage DNLL but inadvertently destroyed cells in other auditory structures. Two rats ( $R 162$ and $R 175)$ received unilateral lesions that dam- 

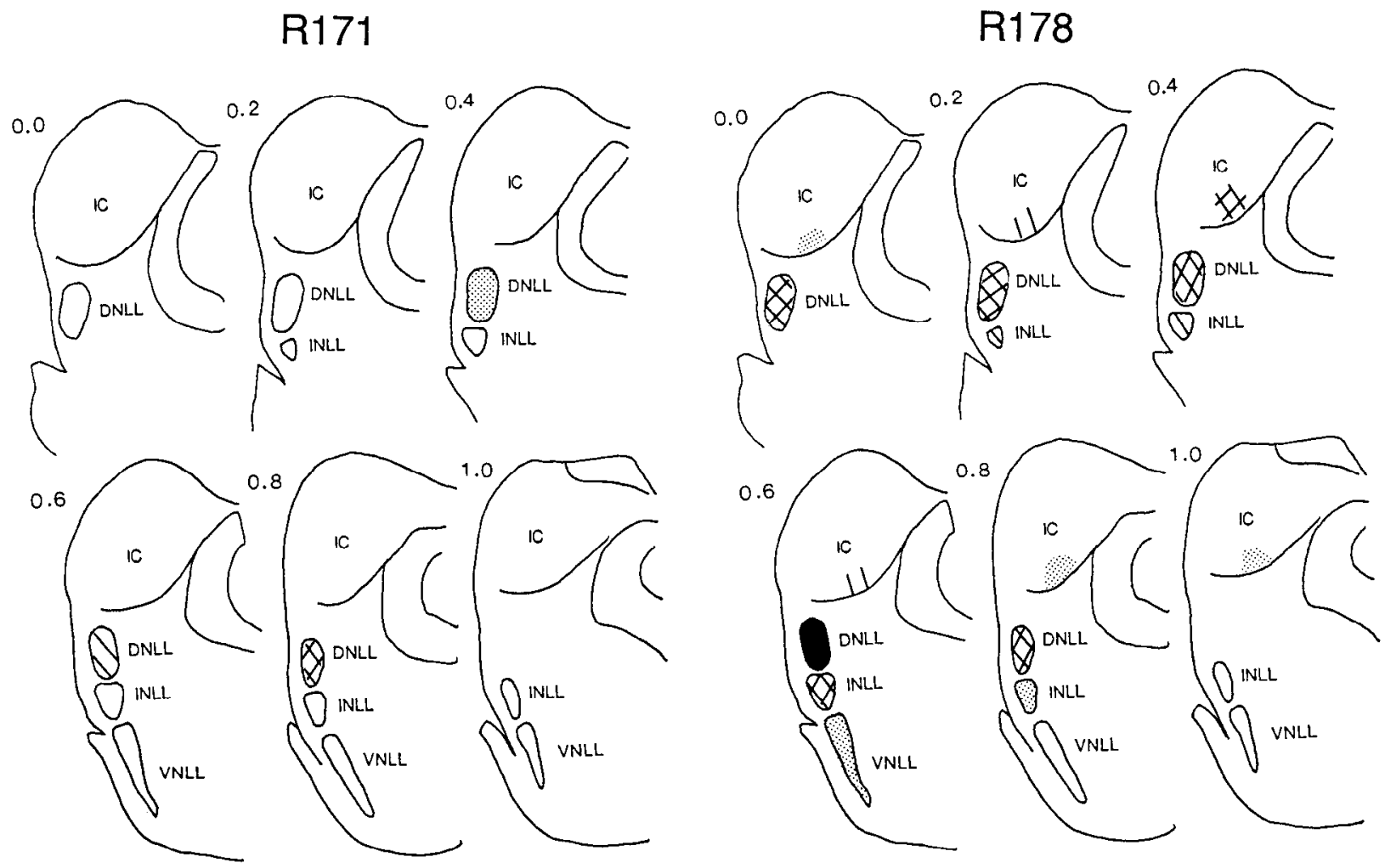

Figure 4. Anatomical reconstructions for two animals (R171 and R178) with left DNLL lesions. Conventions are as in Figure 3.

aged INLL and VNLL on the right side of the brain but caused no cell loss in DNLL. In R162, the damage in INLL ranged from severe to complete and VNLL was completely destroyed. In R175, both INLL and VNLL were completely destroyed. A third rat (R165) sustained minor damage to the IC on the left side of the brain. Cell loss was confined to a small portion of the ventral central nucleus of the IC with no involvement of DNLL. The anatomical reconstructions for these rats are shown in Figure 5.

In three additional cases (R164, R170, and R174), the micropipette was inserted into the DNLL but no kainic acid was injected due to blockage of the pipette tip. No neurological damage was found in any of these cases beyond that produced by the micropipette track. These three cases, together with the three animals with misplaced lesions (R162, R165, and R175), form a lesion control group.

Monaural response thresholds and latencies. Evoked potentials were recorded from primary auditory cortex in both left and right hemispheres of all animals. Prior to systematic tests with binaural clicks, thresholds and latencies were obtained in each hemisphere for responses elicited by a contralaterally presented monaural click. Thresholds, defined as the minimum click intensity (in dB SPL) necessary to elicit an averaged evoked response, were obtained for control animals and animals with DNLL lesions. For normal control cases, the mean thresholds were 43 and $44 \mathrm{~dB}$ for the two hemispheres. For the lesion control group, the mean thresholds were 41 and $42 \mathrm{~dB}$ for the hemispheres ipsilateral and contralateral to the site of pipette insertion. For the DNLL lesion group, the mean thresholds were 33 and $31 \mathrm{~dB}$ for the hemispheres ipsilateral and contralateral to the lesion site.
Although there was a tendency for the mean thresholds to be lower in animals with DNLL lesions than either normal or lesion control cases, the differences were not statistically significant. ANOVA failed to show significant differences due to either lesion effect $[F(2,12)=2.74 ; p>0.05]$, hemisphere $[F(1,12)=$ $0.20 ; p>0.05]$, or hemisphere by lesion interaction $[F(2,12)=$ $0.44 ; p>0.05]$. Comparison of mean thresholds for all groups is shown in Figure 6.

Response latencies were measured from stimulus onset to the first positive peak of the cortical response with click intensity set approximately $40 \mathrm{~dB}$ above threshold. Latencies ranged from 10 to $17 \mathrm{msec}$. For normal control cases, the mean latencies were 14.3 and 14.2 msec for the two hemispheres. For the lesion control group, the mean latencies were 12.6 and $12.7 \mathrm{msec}$ for the hemispheres ipsilateral and contralateral to the pipette track. For the DNLL lesion group, the mean latencies were 13.7 and $13.0 \mathrm{msec}$ for the hemispheres ipsilateral and contralateral to the lesion. ANOVA indicated no significant difference among groups for either the main effect of lesions $[F(2,12)=1.15 ; p>$ $0.05]$, the main effect of hemisphere $[F(1,12)=0.46 ; p>0.05]$, or the interaction term $[F(2,12)=0.49 ; p>0.05]$. The mean latencies for all groups are shown in Figure 7.

Binaural response amplitude. No differences were found among groups or between hemispheres in the maximum evoked response amplitude produced by binaural stimulation. In every case, the largest response was elicited by contralateral leading ipsilateral click pairs. The mean maximum response amplitude was $69 \mu \mathrm{V}$. ANOVA revealed no significant differences among the means for lesion group, hemisphere, or group by hemisphere interaction $[F(2,12)=0.96, p>0.05 ; F(1,12)=0.69, p>0.05$; $F(1,12)=0.89, p>0.05]$. For all further analysis of binaural 
R162

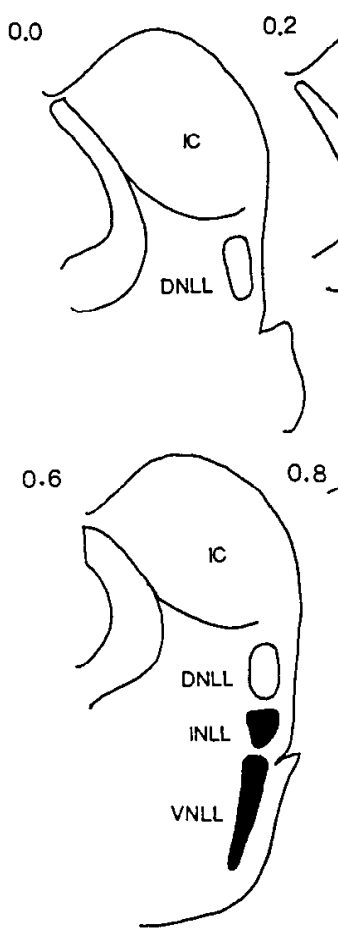

0.4
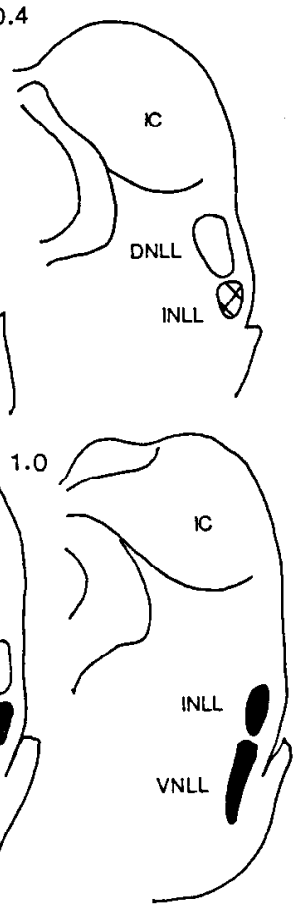

$\mathrm{R} 165$
$\mathrm{R} 175$

$0.0+0.2$
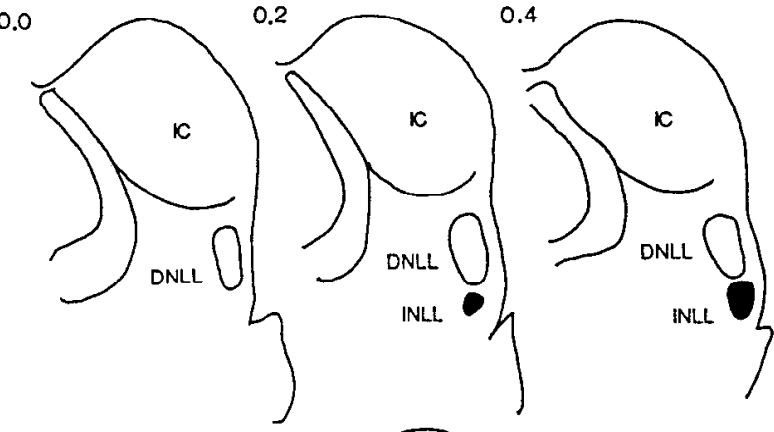

0.6

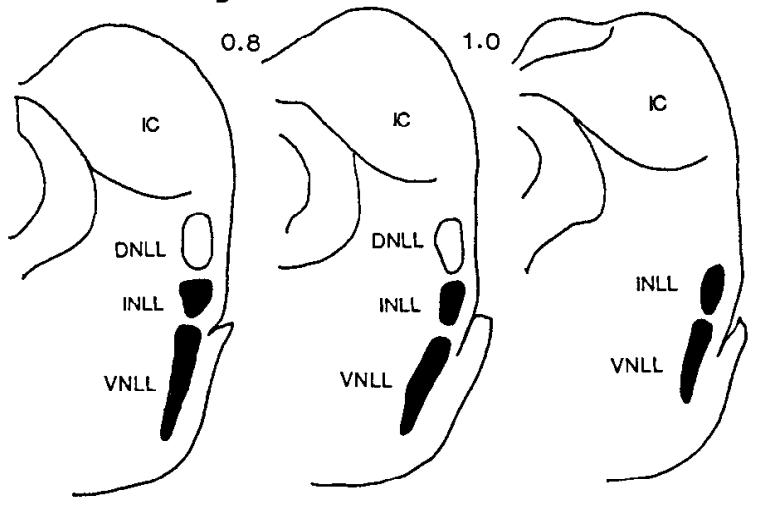

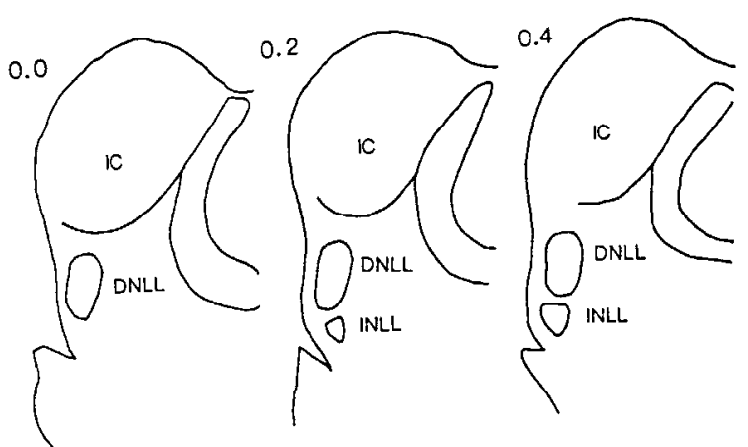
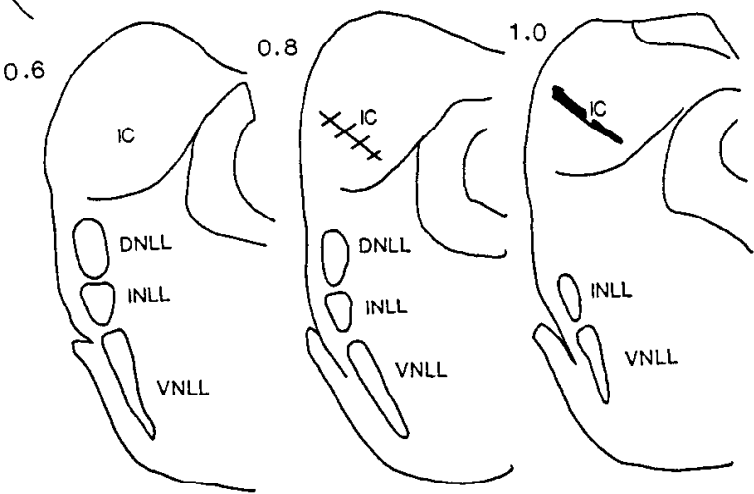

Figure 5. Anatomical reconstructions for three cases with control lesions. In two animals ( $R 162$ and $R 175)$, the lesion destroyed cells in right INLL and VNLL without producing damage in DNLL. In the third case $(R I 65)$, the lesion destroyed cells in the left central nucleus of the IC without damaging the nuclei of the lateral lemniscus. Conventions are as in Figure 3.

data, evoked response amplitudes for each hemisphere were scaled and expressed as a percentage of the maximum for that hemisphere.

In the normal rat, the evoked response amplitude was found to be a sigmoidal function of the ITD between paired clicks delivered to the two ears. Evoked response amplitude was greatest contralateral to the ear that was stimulated first and became progressively reduced as the time difference was shifted in favor of the ipsilateral ear. The ITD functions in the left and right hemispheres tended to be symmetrical mirror images of one 


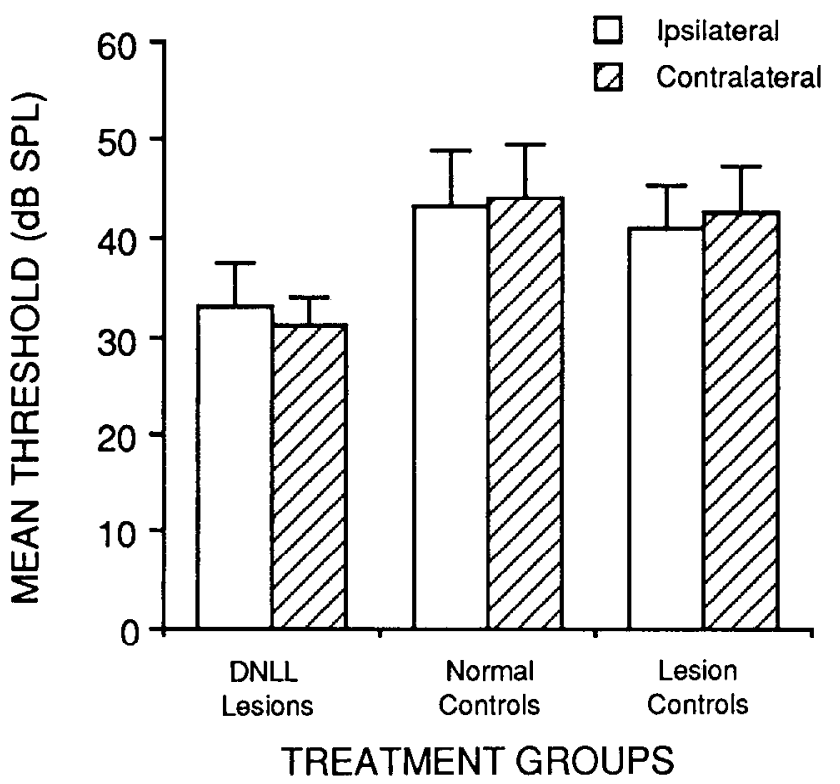

Figure 6. The mean thresholds for eliciting an averaged evoked response in animals with DNLL lesions, animals with control lesions, and normal animals. The thresholds are plotted separately for responses obtained from the hemisphere ipsilateral (open bars) and contralateral (hatched bars) to the lesion. Error bars indicate $1 \mathrm{SE}$ above the mean. There were no significant differences in threshold between hemispheres or among groups.

another, with the two curves intersecting around ITD $=0 \mu \mathrm{sec}$. The slopes of ITD functions in the two hemisphere were similar. The ITD functions for each of the six normal control cases are shown in Figure 8. The evoked response amplitudes were maximum under conditions in which the contralateral click was leading the ipsilateral click by $600 \mu \mathrm{sec}$. Evoked response amplitudes were reduced by roughly $50 \%$ under conditions in which the ipsilateral click was leading the contralateral click by 600 $\mu \mathrm{sec}$. The most pronounced change in amplitude occurred between +250 and $-250 \mu$ sec.

The ITD functions for animals in the lesion control group were similar in every respect to those of normal controls. The ITD curves for each of these six animals are shown in Figure 9. The first two examples were taken from animals with lesions that completely spared DNLL but severely damaged the right INLL and VNLL (R162 and R175). The third was obtained from an animal (R165) with a lesion that destroyed cells in the left ventral IC. The remaining three examples were obtained from animals (R164, R170, and R174) in which the micropipette was inserted into the DNLL without making a kainic acid lesion. In each of these cases, response amplitude was greatest contralateral to the ear that was stimulated first, and was greatly reduced as the binaural time difference was shifted in favor of the ipsilateral ear. The slopes of the functions in the two hemispheres were similar and symmetrical. The amount of reduction in response amplitude was close to $50 \%$ in every case except for R175.

Response amplitude ITD functions were obtained from fivc animals with unilateral DNLL lesions. The curves for each of these animals are presented in Figure 10. The first three examples were from animals with right DNLL lesions (R167, R168, and R173). The other two were from animals with left DNLL lesions (R171 and R178). As can be seen, the ITD functions were markedly asymmetrical with a pronounced reduction

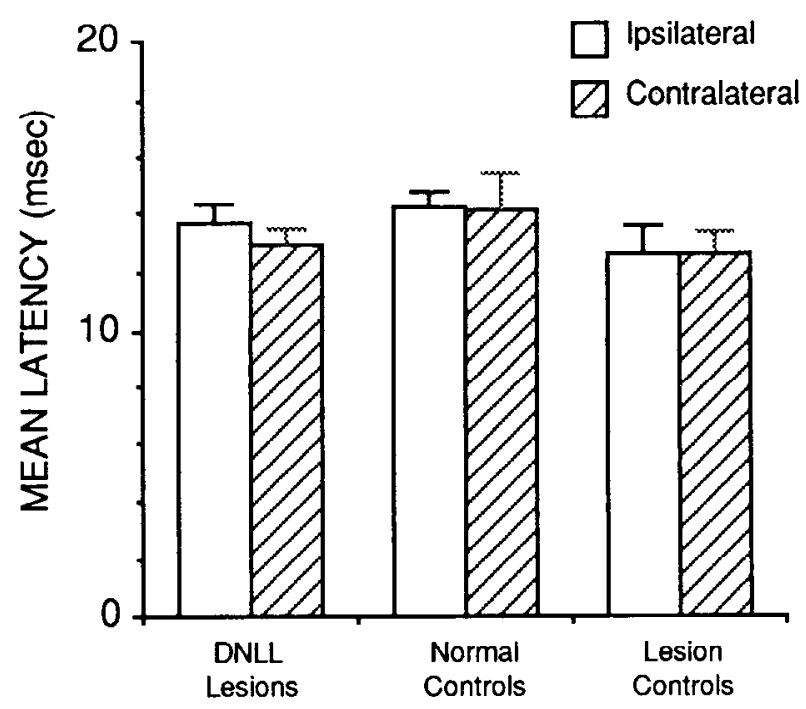

TREATMENT GROUPS

Figure 7. The mean latencies of averaged evoked responses in animals with DNLL lesions, lesion control animals, and normal animals. Latencies of responses from the hemisphere ipsilateral to the lesion are represented by open bars, and latencies from the hemisphere contralateral to the lesion are represented by hatched bars. Error bars indicate $1 \mathrm{SE}$ above the mean. There were no significant differences in latency between hemispheres or among groups.

in the steepness of the slope contralateral to the side of the lesion. The ITD functions from the hemisphere ipsilateral to the lesion were similar to those obtained from normal control cases. In contrast, the functions from the hemispheres contralateral to the lesion were flattened relative to normal and the extent of reduction in response amplitude was much less than expected from controls.

Quantitative analysis of these data was conducted by statistical comparison of the slopes of ITD curves for each of the three groups composed of DNLL lesion, normal control, and lesion control cases. Slopes were defined by the linear best fit of the ITD data between 1250 and $250 \mu$ sec. Responses from the hemisphere ipsilateral and contralateral to the lesion site were treated separately for animals in the DNLL group. For all other animals, cortical responses were parcellated relative to the site of surgical intervention, that is, micropipette placement in the case of the lesion control group and cortical aspiration in the case of the normal controls.

The mean slopes of all ITD curves for each of the three groups are shown in Figure 11. As can be seen, the slope of the curve is greatly reduced only in the hemisphere contralateral to the site of the DNLL lesion. ANOVA indicated that there was a significant effect due to lesion group $[F(2,12)=5.95 ; p<0.05]$, hemisphere $[F(1,12)=14.08 ; p<0.005]$, and hemisphere by lesion group interaction $[F(2,12)=24.54 ; p<0.0001]$. All significant effects were attributed to the reduction in slope in the curves recorded from the hemisphere contralateral to the DNLL lesion. No other significant differences werc found.

\section{Discussion}

The results of the present study lend support to the idea that the DNLL plays an important role in establishing the laterality of binaural responses in the auditory system (Shneiderman et al., 1988; Oliver and Shneiderman, 1989; Shneiderman and 
R169

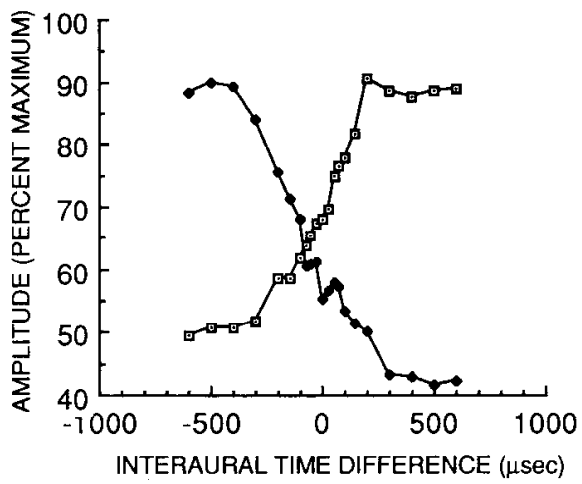

R179

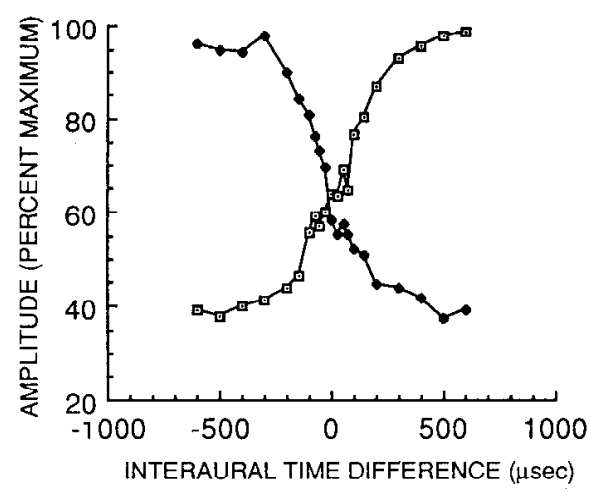

R163

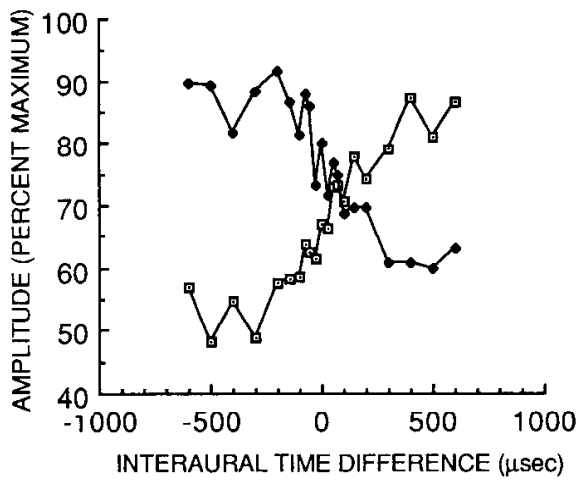

R182

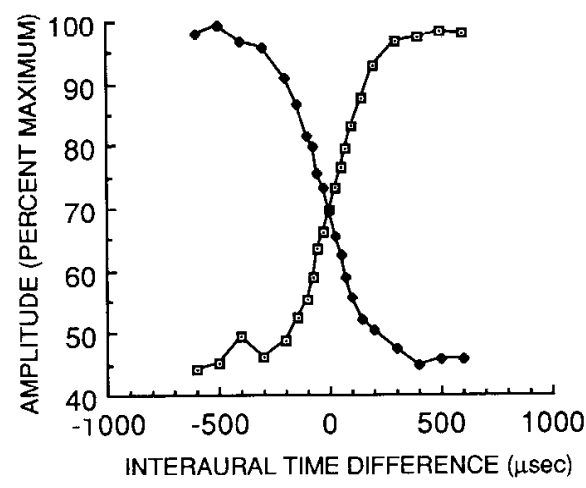

R172

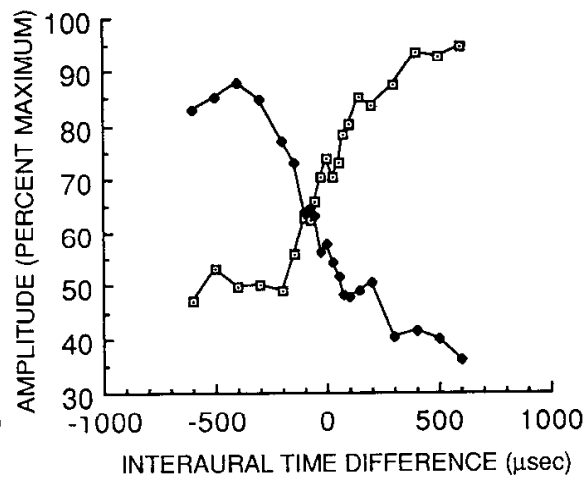

R189

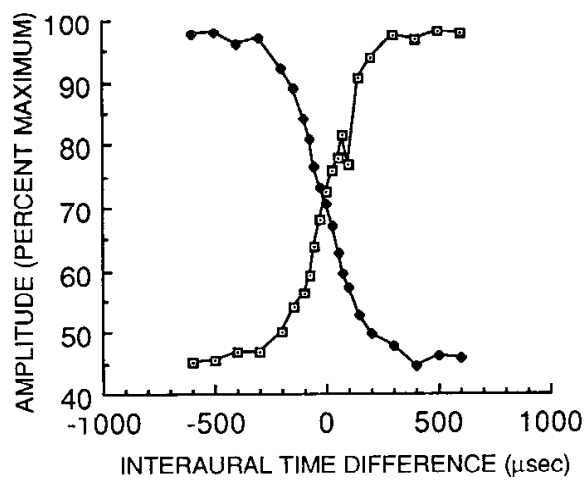

Figure 8. The ITD functions for normal control cases $(R 169, R 163, R 172, R 179, R 182$, and $R 189)$. Response amplitudes are plotted as a function of the ITD for each hemisphere. The amplitudes are expressed as a percentage of the maximum response amplitude obtained during each replication of the ITD sequence. The curves are based on the average of 10 replications. The open squares designate the left hemisphere responses, and solid squares designate the right hemisphere responses. ITDs are expressed in $\mu \mathrm{sec}$ (right ear minus left ear). Thus, positive values indicate right leading left and negative values indicate left leading right click pairs.

Oliver, 1989). Unilateral damage of the DNLL greatly reduced the binaural sensitivity of evoked responses in the auditory cortex contralateral to the lesion, thus weakening the laterality of the response in that hemisphere. The slopes of ITD functions in the hemisphere contralateral to the lesion were flattened relative to normal. As the binaural time difference was shifted progressively in favor of the ipsilateral ear, the evoked response amplitude in normal animals dropped by as much as $50 \%$ in most cases. In contrast, the evoked response amplitude in animals with DNLL lesions was reduced by only $25 \%$. This dramatic difference was not associated with any significant difference in the maximum response amplitude. Rather, the flattened ITD functions in animals with DNLL lesions were due to a reduction in the strength of inhibition produced by stimulation of the ipsilateral ear.

Furthermore, unilateral DNLL lesions had no significant effect on monaural response thresholds or response latencies of evoked responses in either hemisphere. Thus, abnormal ITD functions cannot be attributed to alterations in absolute sensitivity or changes in monaural processing. The flattened ITD functions reflect, specifically, a disruption of binaural processing in the central auditory system.

The abnormal binaural response functions are probably caused by a reduction or elimination of inhibitory projections from DNLL to either IC or DNLL on the opposite side of the brain. This interpretation is consistent with both neuroanatomical and neurochemical studies of DNLL efferent projections. Immu- nocytochemistry has shown that most of the neurons in DNLL react positively for the inhibitory neurotransmitter GABA or its related enzyme glutamic acid decarboxylase (Adams and Mugnaini, 1984; Thompson et al., 1985; Moore and Moore, 1987; Robcrts and Ribak, 1987; Glendenning and Baker, 1988). Electron microscopic investigations combined with anterograde transport techniques indicate that more than $90 \%$ (perhaps all) of the DNLL terminals in the contralateral IC have flattened or pleomorphic synaptic vesicles characteristic of inhibitory neurons (Shneiderman and Oliver, 1989). A similar projection has been described from DNLL to the contralateral DNLL (Oliver and Shneiderman, 1989). It is likely that both projections are GABAergic. Also, unilateral kainic acid lesions of the DNLL greatly reduce the in vitro electrically evoked release of GABA in the IC contralateral to the lesion (Potashner et al., 1991). Therefore, the most likely interpretation of our physiological data is that unilateral kainic acid lesions of the DNLL eliminate or reduce GABAergic inhibition in the auditory midbrain contralateral to the lesion and that this effect is reflected in the amplitude of evoked responses recorded from primary auditory cortcx.

DNLL lesions had no obvious effect on binaural responses recorded from the auditory cortex in the ipsilateral hemisphere. The lack of effect is significant in light of the fact that the DNLL does have an ipsilateral projection to the IC. Two considerations may have bearing on the lack of physiological effect. First, unlike the contralateral projection, the ipsilateral projection from DNLL 
R162

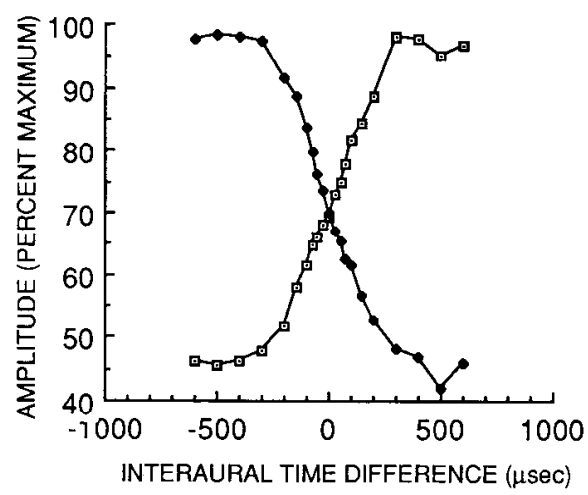

R164

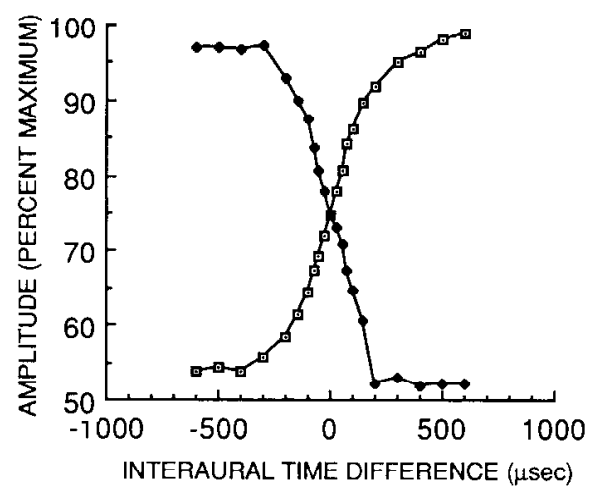

R175

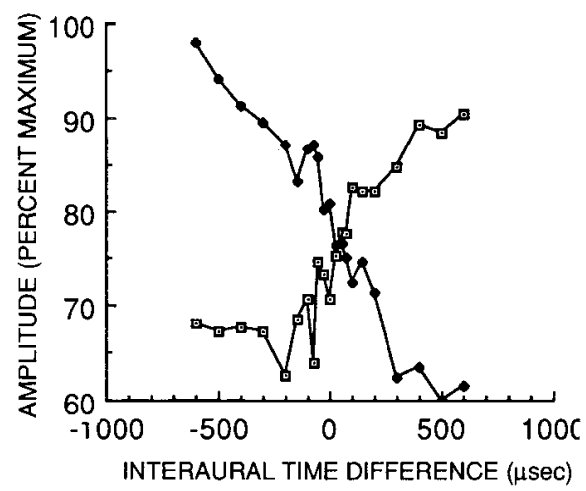

R170

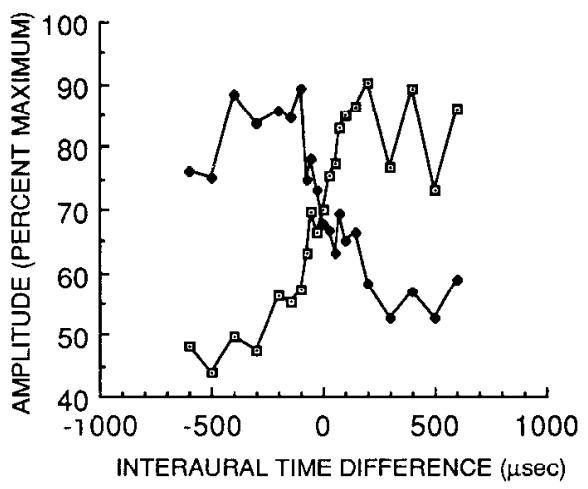

R165

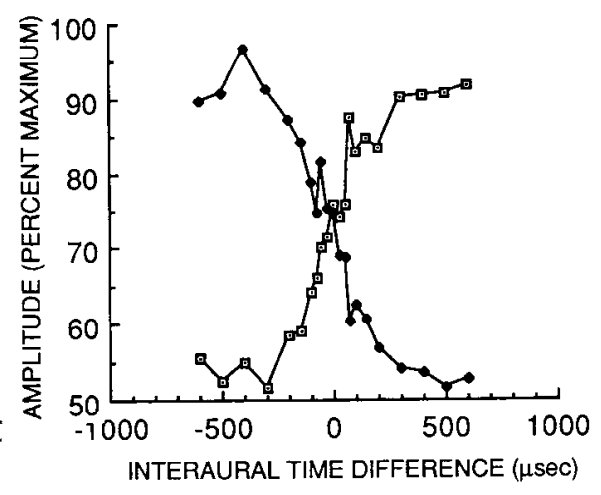

R174

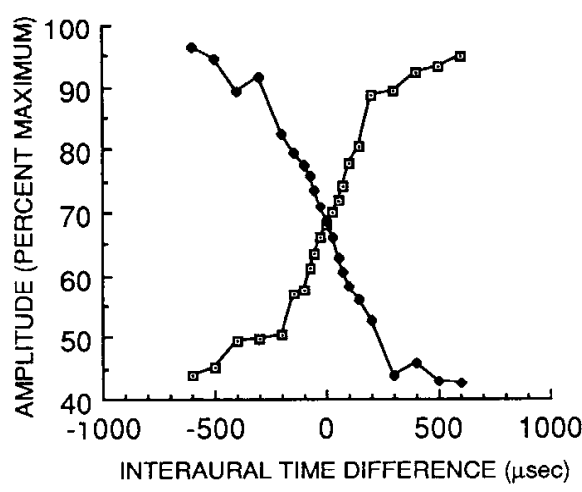

Figure 9. The ITD functions for lesion control animals $(R 162, R 175, R 165, R 164, R 170$, and $R 174)$. Two animals $(R 162$ and $R 175)$ had extensive damage to the right INLL and VNLL. One animal $(R 165)$ had moderate cell loss in the right $I C$. The other animals had no damage beyond that produced by introduction of a micropipette into the lateral lemniscus. Symbols are as in Figure 8.

to IC is not exclusively associated with inhibitory terminals, but has both an excitatory and inhibitory component. Following injection of an anterograde tracer into DNLL, a significant number of axon terminals in ipsilateral IC are characterized by rounded synaptic vesicles usually associated with excitatory transmitters. The density of terminals with pleomorphic vesicles is much less in ipsilateral than in contralateral IC (Shneiderman and Oliver, 1989). Also, the effect of unilateral DNLL lesions on electrically evoked release of GABA in vitro is less pronounced in ipsilateral than in contralateral IC (Potashner et al., 1991). Thus, the ipsilateral inhibitory projection to IC may be less effective than the contralateral projection. Second, the ipsilateral and contralateral projections to IC arise from separate populations of neurons in DNLL. Only a small percentage of the neurons in DNLL have collaterals to both sides of the brain (Hutson et al., 1991). Thus, the separate populations of ipsilaterally and contralaterally projecting neurons may be driven by different synaptic input or activated under quite different stimulus conditions. It is highly probable, based on the anatomical segregation of the cells involved, that the ipsilateral and contralateral projections serve different physiological functions. Therefore, the ipsilateral projection may make little or no contribution to binaural responses in auditory cortex at least under the conditions employed in this study.

The predominantly contralateral effect of DNLL lesions has been confirmed recently by single-unit recordings directly from the central nucleus of the IC (Li and Kelly, 1992). In this study, neural activity in DNLL was temporarily blocked by local injection of the glutamate antagonist, kynurenic acid. Unilateral blockade resulted in a partial release from inhibition and a reduction in the slope of interaural intensity difference (IID) functions contralateral to the injection, but produced no discernible change in IID functions ipsilateral to the injection. This effect was probably due to elimination of the GABAergic inhibitory projection from DNLL to the contralateral DNLL or IC.

Although unilateral lesions of DNLL reduced the slope of evoked response ITD functions in the contralateral hemisphere, the slope was not reduced to zero. This result may be related to the completeness of the DNLL lesions. In most cases presented here, the lesions produced incomplete cell loss in DNLL. On the other hand, some evidence of binaural interaction was found even in animals with severe or nearly complete lesions (e.g., R178). Other sources of binaural interaction might have contributed to cortical responses even in the complete absence of cells in DNLL. For example, it is well known that the nuclei of the superior olivary complex (SOC) are involved in processing binaural time and intensity differences. Binaural interactions are established in the SOC prior to any further processing in the DNLL (Boudreau and Tsuchitani, 1968; Goldberg and Brown, 1969; Tsuchitani and Boudreau, 1969; Guinan et al., 1972a,b; Harnischfeger et al., 1975; Inbody and Feng, 1981; Caird and Klinke, 1983; Yin and Chan, 1990). Therefore, the binaural responses that remain following a unilateral DNLL lesion may be a reflection of binaural interactions in the SOC. The excitatory output of neurons in SOC almost certainly influences activity in the IC through pathways that completely bypass the DNLL. Furthermore, the lateral superior olive (LSO) 
R167

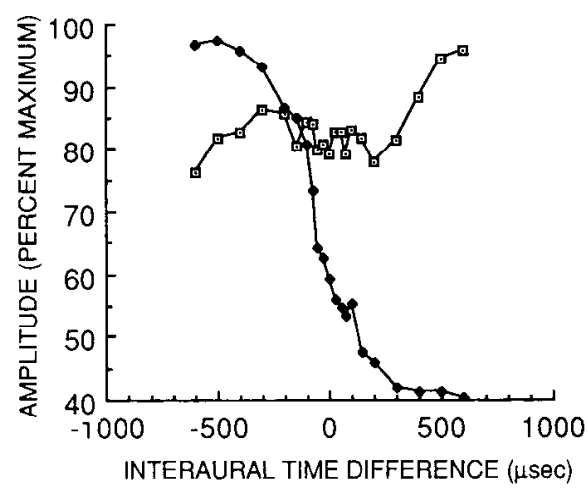

R168

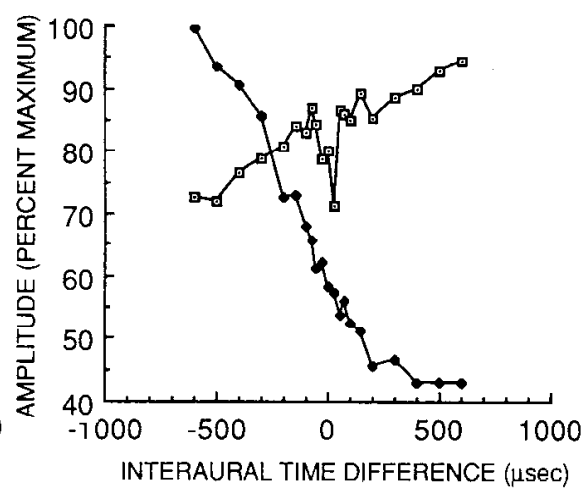

R173

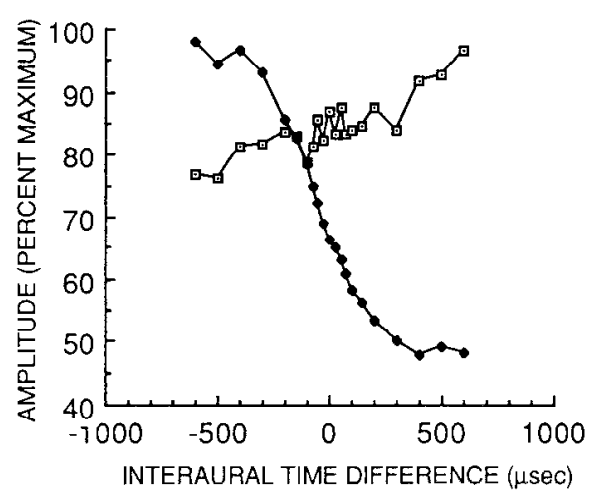

R171

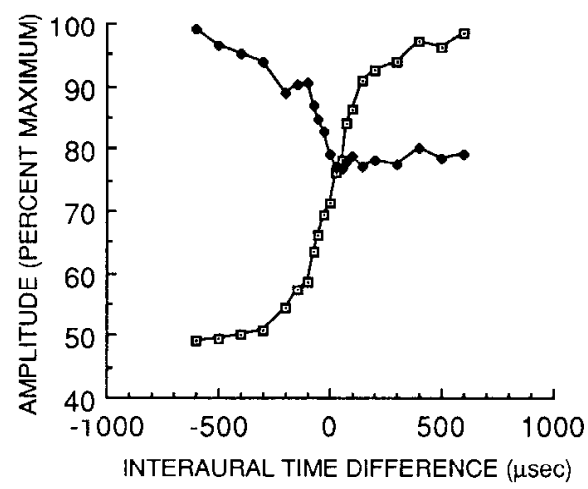

R178

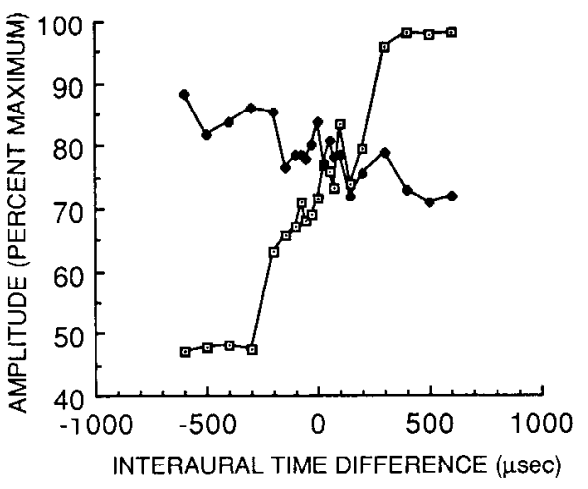

Figure 10. The ITD functions for three animals with right DNLL $(R 167, R I 68$, and $R 173)$ and two animals with left DNLL lesions $(R 171$ and $R 178$ ). The slope of the ITD function was reduced in every case in the hemisphere contralateral to the lesion. Symbols are as in Figure 8.

is the source of an extensive inhibitory glycinergic projection to the ipsilateral IC (Hutson et al., 1987; Saint Marie et al., 1989; Saint Marie and Baker, 1990). This projection would be expected to have a physiological effect on binaural responses similar to that proposed for GABAergic projections from DNLL, namely, an inhibition of binaural responses under conditions in which ipsilateral stimulation predominates over contralateral stimulation. Both the ipsilateral glycinergic projection from LSO and the contralateral GABAergic projection from DNLL might contribute to the suppression of cortical evoked responses by ipsilateral sound sources.

Damage to the INLL and VNLL had no effect on binaural evoked responses recorded in auditory cortex of either hemisphere. This result is consistent with the anatomical connections and physiological responses in these nuclei. Tract tracing studies indicate that neither of these nuclei receive substantial binaural input. The VNLL receives an overwhelming majority of its afferent input from the contralateral ventral cochlear nucleus, and INLL receives its afferent projections from the contralateral cochlear nucleus and the medial nucleus of the trapezoid body, which is itself contralaterally innervated (Glendenning et al., 1981; Warr, 1982; Zook and Casseday, 1982). The cells in VNLL and INLL are primarily driven by contralateral monaural rather than binaural stimulation (Aitkin et al., 1970; Brugge et al., 1970; Covey and Casseday, 1986, 1991). Although the possibility cannot be ruled out that either or both of these nuclei might make some contribution to binaural responses through connections with more rostral structures, the results of the present study provide no indication of involvement in binaural

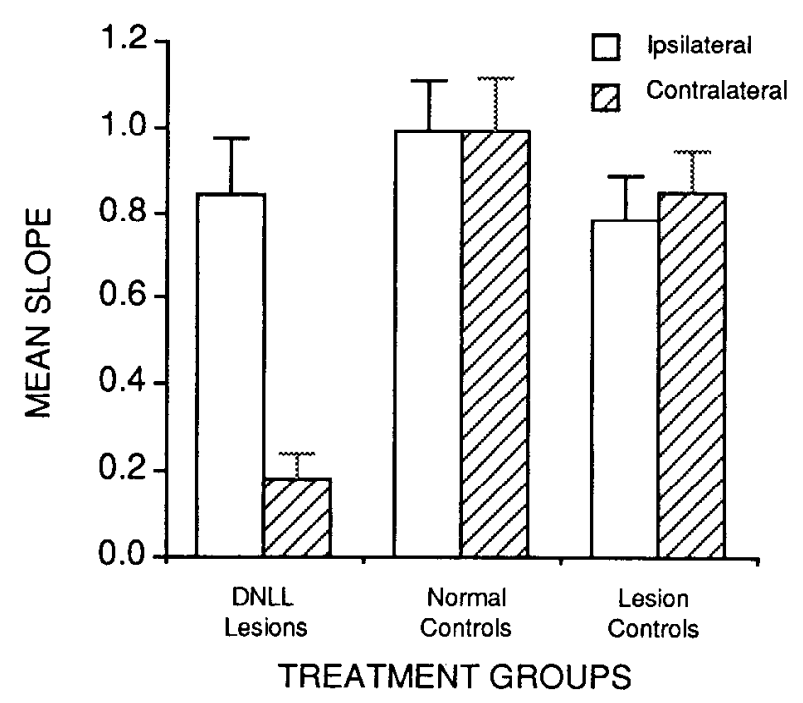

Figure I1. The mean slopes derived from the ITD functions in both hemispheres plotted for the DNLL lesion group, the lesion control group, and the normal group. The slopes obtained from the hemisphere ipsilateral to the DNLL lesion, pipette placement, or cortical extirpation are shown as open bars. The slopes obtained from the hemisphere contralateral to these treatments are shown as hatched bars. Error bars indicate $1 \mathrm{SE}$ above the mean. The mean slope obtained from the hemisphere contralateral to the DNLL lesion was significantly reduced relative to that obtained under any other condition. 
processing. It is possible that these nuclei serve exclusively monaural auditory functions.

In conclusion, our data support the concept of multiple levels of binaural interaction in the auditory pathway. The SOC provides an early opportunity for binaural processing. The neurons in both the LSO and the medial superior olive (MSO) are sensitive to binaural input and respond selectively to ITDs and/or IIDs. In the LSO, most cells are excited by ipsilateral stimulation and are inhibited by contralateral stimulation, thus showing a strong preference for sounds located in the ipsilateral acoustic field. In the MSO, many cells are excited by stimulation of either ear alone and are facilitated by combined binaural stimulation. Others are excited by stimulation of one ear and are inhibited by stimulation of the opposite ear. The maximum firing of neurons in MSO is typically produced by binaural input associated with contralateral field positions. The rostral excitatory projections of LSO and MSO converge to provide a predominantly contralateral representation of space in the DNLL and central nuclcus of the IC (Glendenning and Masterton, 1983; Glendenning et al., 1985; Saint Marie et al., 1989).

The DNLL plays a role in shaping binaural responses in more rostral structures by reinforcing the imbalance in neural activity already established on the two sides of the brain. The contribution of DNLL might, in part, be a reflection of parallel processing independent of the SOC. Many neurons in DNLL (about $10 \%$ in the cat) are driven monaurally by sounds presented to the contralateral ear, probably through a direct connection from the opposite cochlear nucleus (Aitkin et al., 1970). These neurons likely form part of the inhibitory pathway from DNLL to the DNLL and IC on the opposite side of the brain. This inhibitory projection from DNLL could converge with a direct contralateral excitatory projection from cochlear nucleus to IC to establish binaural interactions within the IC itself. Consistent with this possibility is the finding that kainic acid lesions of the SOC do not completely eliminate binaural responses in IC (Kelly and Li, 1992; Sally and Kelly, 1992). Also, the DNLL might play a role in lateralization through hierarchical processing of binaural responses that originate in the SOC. One of the major projections to the DNLL comes from the SOC, which has already provided an initial stage of binaural processing. In either case, the increased activity in DNLL associated with a contralateral sound source would exert a net inhibitory effect on DNLL and IC on the opposite side of the brain. This "lateral inhibition" produced by the crossed GABAergic projection from DNLL would have the effect of maintaining or enhancing the laterality of binaural responses in the auditory pathway.

\section{References}

Adams JC (1979) Ascending projections to the inferior colliculus. J Comp Neurol 183:195-538.

Adams JC, Mugnaini E (1984) Dorsal nucleus of the lateral lemniscus: a nucleus of GABAergic projection neurons. Brain Res Bull 13:585590.

Aitkin LM, Anderson DJ, Brugge JF (1970) Tonotopic organization and discharge characteristics of single neurons in nuclei of the lateral lemniscus of the cat. J Neurophysiol 33:421-440.

Beyerl BD (1978) Afferent projections to the central nucleus of the inferior colliculus in the rat. Brain Res 145:209-223.

Boudreau JC, Tsuchitani C (1968) Binaural interaction in the cat superior olive S segment. J Neurophysiol 31:442-454.

Brugge JF, Anderson DJ, Aitkin LM (1970) Responses of neurons in the dorsal nucleus of the lateral lemniscus of the cat to binaural tonal stimulation. J Neurophysiol 33:441-458.

Brunso-Bechtold JK, Thompson GC, Masterton RB (1981) HRP study of the organization of auditory afferents ascending to the central nucleus of the inferior colliculus in cat. J Comp Neurol 197:705-722.

Caird D, Klinke R (1983) Processing of binaural stimuli by cat superior olivary complex neurons Exp Brain Res 52:385-399.

Coleman JR, Clerici WJ (1987) Source of projections to subdivisions of the inferior colliculus in the rat. J Comp Neurol 262:215-226.

Covey E, Casseday JH (1986) Connectional basis for frequency representation in the nuclei of the lateral lemniscus of the bat Eptesicus fuscus. J Neurosci 6:2926-2940.

Covey E, Casseday JH (1991) The monaural nuclei of the lateral lemniscus in an echolocating bat: parallel pathways for analyzing temporal features of sound. J Neurosci 11:3456-3470.

Faingold CL, Gehlbach G, Caspary DM (1989) On the role of GABA as an inhibitory neurotransmitter in inferior colliculus neurons: iontophoretic studies. Brain Res 500:302-312.

Faingold CL, Boersma Anderson CA, Caspary DM (1991) Involvement of GABA in acoustically-evoked inhibition in inferior colliculus neurons. Hearing Res 52:201-216.

Glendenning KK, Baker BN (1988) Neuroanatomical distribution of receptors for three potential inhibitory neurotransmitters in the brainstem auditory nuclei of the cat. J Comp Neurol 275:288-308.

Glendenning KK, Masterton RB (1983) Acoustic chiasm: efferent projections of the latcral supcrior olive. J Neurosci 3:1521-1537.

Glendenning KK, Brunso-Bechtold JK, Thompson GC, Masterton RB (1981) Ascending auditory afferents to the nuclei of the lateral lemniscus. J Comp Neurol 197:673-703.

Glendenning KK, Hutson KA, Nudo RJ, Masterton RB (1985) Acoustic chiasm. II. Anatomical basis of binaurality in lateral superior olive of cat. J Comp Neurol 232:261-285.

Goldberg JM, Brown PB (1969) Response of binaural neurons of dog superior olivary complex to dichotic tonal stimuli: some physiological mechanisms of sound localization. J Neurophysiol 32:613-636.

Goldberg JM, Moore RY (1967) Ascending projections of the lateral lemniscus in the cat and monkey. J Comp Neurol 129:143-156.

Guinan JJ Jr, Guinan SS, Norris BE (1972a) Single auditory units in the superior olivary complex. I. Responses to sounds and classifications based on physiological properties. Int J Neurosci 4:101-120.

Guinan JJ Jr, Norris BE, Guinan SS (1972b) Single auditory units in the superior olivary complex. II. Locations of unit categories and tonotopic organization. Int $J$ Neurosci 4:147-166.

Harnischfeger G, Neuweiler G, Schlegel P (1975) Interaural time and intensity coding in superior olivary complex and inferior colliculus of the echolocating bat Molossus ater. J Neurophysiol 53:89-109.

Hutson KA, Glendenning KK, Masterton RB (1987) Biochemical basis for the acoustic chiasm? Soc Neurosci Abstr 13:548.

Hutson KA, Glendenning KK, Masterton RB (1991) Acoustic chiasm IV: eight midbrain decussations of the auditory system. J Comp Neurol 312:105-131.

Inbody SB, Feng AS (1981) Binaural response characteristics of single neurons in the medial superior olivary nucleus of the albino rat. Brain Res 210:361-366.

Kelly JB, Li L (1992) Response properties of neurons in the rat's inferior colliculus following kainic acid lesions of the superior olivary complex: interaural intensity difference functions. Assoc Res Otolaryngol Abstr 15:61.

Kudo M (1981) Projections of the nuclei of the lateral lemniscus in the cat: an autoradiographic study. Brain Res 221:57-69.

Li L, Kelly JB (1992) A reversible block of neural activity in DNLL alters binaural responses in contralateral inferior colliculus. Assoc Res Otolaryngol Abstr 15:62.

Moore JK, Moore RY (1987) Glutamic acid decarboxylase-like immunoreactivity in brainstem auditory nuclei of the rat. J Comp Neurol 260:157 -174.

Oliver DL, Shneiderman A (1989) An EM study of the dorsal nucleus of the lateral lemniscus: inhibitory, commissural, synaptic connections between ascending auditory pathways. J Neurosci 9:967-982.

Potashner SJ, Shneiderman A, Chase MB, Benson C, Rockwood JM (1991) GABA and glycine release from guinea pig inferior colliculus after ablation of dorsal nucleus of lateral lemniscus. Soc Neurosci Abstr 17:00.

Roberts RC, Ribak CE (1987) GABAergic neurons and axon terminals in the brainstem auditory nuclei of the gerbil. J Comp Neurol 258: 267-280.

Saint Marie RL, Baker RA (1990) Neurotransmitter-specific uptake of $\left[{ }^{3} \mathrm{H}\right] \mathrm{glycine}$ from the inferior colliculus by ipsilateral projections of 
the superior olivary complex and nuclei of the lateral lemniscus. Brain Res 524:244-253.

Saint Marie RL, Ostapoff EM, Morest DK, Wenthold RJ (1989) Glycine-immunoreactive projection of the cat lateral superior olive: possible role in midbrain ear dominance. J Comp Neurol 279:382-396.

Sally SL, Kelly JB (1992) Effects of superior olivary complex lesions on binaural responses in rat inferior colliculus. Brain Res 572:5-18.

Shneiderman A, Oliver DL (1989) EM autoradiographic study of the projections from the dorsal nucleus of the lateral lemniscus: a possible source of inhibitory inputs to the inferior colliculus. J Comp Neurol 286:28-47.

Shneiderman A, Oliver DL, Henkel CK (1988) Connections of the dorsal nucleus of the lateral lemniscus: an inhibitory parallel pathway in the ascending auditory system? J Comp Neurol 276:188-208.

Thompson GC, Cortez AM, Lam DM (1985) Localization of GABA immunoreactivity in the auditory brainstem of guinea pigs. Brain Res 339:119-122.
Tsuchitani C, Boudreau JC (1969) Stimulus level of dichotically presented tones and cat superior olive S-segment cell discharge. J Acoust Soc Am 46:978-988.

Warr WB (1982) Parallel ascending pathways from the cochlear nucleus: neuroanatomical evidence of functional specialization. In: Contributions to sensory physiology, Vol 7 (Neff WD, ed), pp 1-38. New York: Academic.

Yin TCT, Chan JCK (1990) Interaural time sensitivity in medial superior olive of cat. J Neurophysiol 64:465-488.

Zook JM, Casseday JH (1982) Origin of ascending projections to the inferior colliculus in the mustache bat, Pteronotus parnelli. J Comp Neurol 207:14-28.

Zook JM, Casseday JH (1987) Convergence of ascending pathways at the inferior colliculus of the mustache bat, Pteronotus parnelli. J Comp Neurol 261:347-361. 